initial presentation of chronic myelogenous leukemia, medullary chronic phase in era of tyrosine kinase inhibitors: a report of 11 cases. Am J Hematol 2015;90:E146-8.

5. Dasappa L, Thanky AH, Kuntegowdanahalli LC, et al. Myeloid sarcoma as the first sign of progression of chronic myeloid leukemia in medullary chronic phase: experience from a Tertiary Cancer Centre in Southern India. Gulf J Oncolog 2017;1:21-5.

6. Chen Z, Wang W, Cortes JE, et al. Differential clinical and prognostic impact of myeloid sarcoma vs medullary myeloid blast phase of chronic myelogenous leukemia in the era of tyrosine kinase inhibitor therapy. Blood Cancer J 2016;6:e418.

7. Samborska M, Derwich K, Skalska-Sadowska J, Kurzawa P, Wachowiak J. Myeloid sarcoma in children - diagnostic and therapeutic difficulties. Contemp Oncol (Pozn) 2016;20:444-8.

\section{An interesting story of a clone}

TO THE EDITOR: Aplastic anemia (AA) is a hematological disorder that leads to significant mortality and morbidity. Clonal evolution in AA after immunosuppressive therapy (IST) is a major concern. Since Dameshek's 1967 editorial in Blood, titled "Riddle: what do aplastic anemia, paroxysmal nocturnal hemoglobinuria (PNH) and 'hypoplastic leukemia' have in common?," to genomic data have increased our understanding of clonal evolution; however, our knowledge remains incomplete $[1,2]$. The earliest evidence came from reports of the clustering of myelodysplastic syndrome (MDS) and $\mathrm{PNH}$ in known cases of AA. Many case reports, series, and structured genetic studies have provided evidence of clonal hematopoiesis $(>50 \%)$ and the development of clonal diseases in AA. Complications of clinically relevant $\mathrm{PNH}$ have been described in 15-25\% of patients with accquired AA treated by IST. However, MDS and acute myeloid leukemia (AML) are reported in $5-15 \%$ of AA cases within $5-11$ years [3]. The significantly increased risk of clonal evolution in the context of inherited bone marrow failure disorder (IBMF) is attributed to the nature of the genetic lesions of these syndromes [2].
Philadelphia positivity is a hallmark of chronic myeloid leukemia (CML) and well described in acute lymphocytic/lymphoblastic leukemia (ALL). BCR-ABL positivity in AML has been investigated and raised doubts regarding CML in blast crisis [4]. With emerging data, the World Health Organization (WHO) described Philadelphia ( $\mathrm{Ph}$ )/ $B C R$ - $A B L$-positive AML in 2016 as a provisional entity under the heading "AML with recurrent cytogenetic abnormalities" [5]. $\mathrm{Ph}$ positivity in AML is a rare event and usually falls in one of the three types: AML-not otherwise specified, AML with myelodysplasia-related changes, and core-binding factor (CBF) AML, with $\mathrm{Ph}$ as an additional abnormality. A subset of Ph-positive AML has been reported during the evolution in known cases of MDS [6]. However, to the best of our knowledge, the development of Philadelphiapositive AML in AA has not been reported.

We hereby report this unusual case of clonal evolution to Philadelphia-positive AML in a case of AA on the wheels of $\mathrm{PNH}$.

\section{Case}

A 17-year-old woman presented to our outpatient department in 2010 with chief complaints of fatigue and intermittent fever for 1.5 months. She also had a 1-week history of excessive bleeding per vaginum. Examination revealed petechiae on her hands and legs. Organomegaly, lymphadenopathy, and stigmata of IBMF were not observed. Complete blood count (CBC) analysis revealed pancytopenia (hemoglobin, $9 \mathrm{~g} / \mathrm{dL}$; total leukocyte count, $2.5 \times 10^{9} / \mathrm{L}$; absolute neutrophil count, $0.4 \times 10^{9} / \mathrm{L}$; platelet count, $5 \times 10^{9} / \mathrm{L}$ ). Peripheral blood smear showed predominantly mature-appearing lymphocytes with normocytic normochromic RBCs. No blasts or abnormal cells were noted. Her corrected reticulocyte count was $0.2 \%$. Serologic testing for hepatitis B surface antigen ( $\mathrm{HBsAg}$ ) and hepatitis $\mathrm{C}$ virus ( $\mathrm{HCV}$ ) was negative.

Bone marrow aspirate showed fat-rich marrow fragments with a paucity of normal hematopoietic elements. Biopsy (Fig. 1A) revealed a hypocellular marrow, with overall cellularity of less than $5 \%$. Stress cytogenetic testing showed no difference in sensitivity to mitomycin $C$ between the patient and the control. Peripheral blood smear showed a

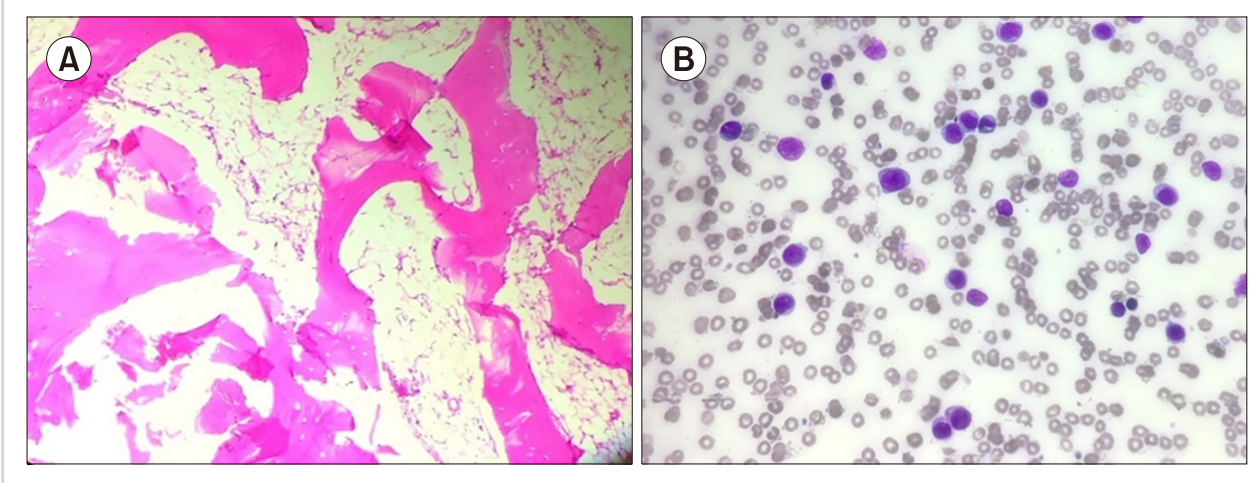

Fig. 1. Hematoxylin and eosin-stained bone marrow biopsy section $(\times 10)$ from the year 2010 showing hypocellular marrow, with overall cellularity of less than $5 \%(\mathrm{~A})$. A Jenner and Giemsa-stained bone marrow aspirate smear $(\times 10)$ from the year 2018 showing more than $90 \%$ blasts (B). 
normal karyotype of $46 \mathrm{XX}$. PNH testing by multiparametric flow cytometry (FCM) fluorescent aerolysin (FLAER)-based assay (sensitivity $1 \%$ ) showed absence of $\mathrm{PNH}$ clones. A final diagnosis of severe AA was made after ruling out secondary causes of myelosuppression.

The patient was started on oral IST (cyclosporine and anabolic steroids) and counseled to receive bone marrow transplant as the treatment of choice. Due to resource constraints, the patient received oral immunosuppressive therapy. She was transfusion-independent for 4 years and showed partial response to therapy. The patient again presented to the outpatient department with anemia symptoms in June 2017, with no history suggestive of bleeding or recurrent infection. Examination revealed no organomegaly. A routine workup with $\mathrm{PNH}$ assay was ordered. The FCM-FLAER-based assay showed 59\% PNH clones in granulocytic series and $47.3 \%$ clones in monocytic series. The patient's cytopenia continued to worsen with no response to treatment. Repeat PNH testing was advised. PNH testing was performed in January 2018 using CD45, FLAER, CD15, CD24, CD64, and CD14 on a multiparametric FCM platform. CD45 side-scatter (Ssc) dot plots showed a cluster of events in CD45 dim and a moderate side-scatter area in the blast region. Peripheral smear examination of the same sample revealed $25 \%$ blasts and no basophils. Bone marrow evaluation showed $>90 \%$ blasts (Fig. 1B), which were positive for non-specific esterase and myeloperoxidase stain on cytochemistry analysis. FCM immunophenotyping of the blasts showed positivity for CD13, CD33, CD34, HLA-DR, MPO, CD64, and CD11c, suggesting AML with monocytic differentiation. Cytogenetic analysis of the bone marrow revealed $\mathrm{t}(9 ; 22)(\mathrm{q} 34 ; \mathrm{q} 11)$ and $\mathrm{t}(7 ; 17)(\mathrm{q} 22: \mathrm{q} 21)$ in all 20 metaphases (Fig. 2). The patient showed no splenomegaly at this presentation. A final diagnosis of clonal evolution to $\mathrm{Ph}$-positive AML in a known case of AA was made. The patient had febrile neutropenia and was treated with broad-spectrum antibiotics and antifungal agents. She denied any chemotherapy and left against medical advice. The patient has since been lost to follow-up.

\section{Discussion}

The exhaustion of hematopoietic stem cells by immunological attack leads to pancytopenia in acquired AA. Immunological escape by glucosyl phosphatidylinositol (GPI) anchor or human leukocyte antigen (HLA)-deficient cells gives them a survival advantage. This is a possible explanation for the $>50 \%$ of AA cases showing clonal hematopoiesis in the form of tiny PNH clones on FCM, chromosome $6 \mathrm{p}$ uniparental disomy on single nucleotide polymorphism (SNP) array karyotyping, or positivity for mutations [3]. The detection of PNH clones has clinical utility, as their presence predicts a better response to IST and serves as a surrogate marker to rule out $\operatorname{IBMF}[2,7]$. Clinically significant diatheses including $\mathrm{PNH}, \mathrm{MDS}$, or $\mathrm{AML}$ are seen in only $15-25 \%$ of acquired AA cases. The exact mechanism for this observation is still not completely understood. The high risk of clonal evolution in IBMF is attributed to differences in the basic pathogenesis of these disorders.

The evolution of AA to AML is very well described [3]. The Philadelphia chromosome in the context of AA is described in rare case reports at baseline or on evolution to ALL or CML in the literature [8, 9]. Philadelphia-positive AML is itself a very rare entity. To the best of our knowledge, this is the first described case of Ph-positive AML in a known case of AA. A review of Ph-positive AML literature showed that many cases evolved in known cases of MDS or harbored additional MDS-defining cytogenetic abnormalities to become AML with myelodysplasia-related changes as per the recent WHO classification $[4,10,11]$. De novo MDS cases harboring Philadelphia chromosome at baseline have also been reported [6]. The significant overlap between $\mathrm{AA}, \mathrm{PNH}, \mathrm{MDS}$, and AML may explain the $\mathrm{Ph}$ positivity

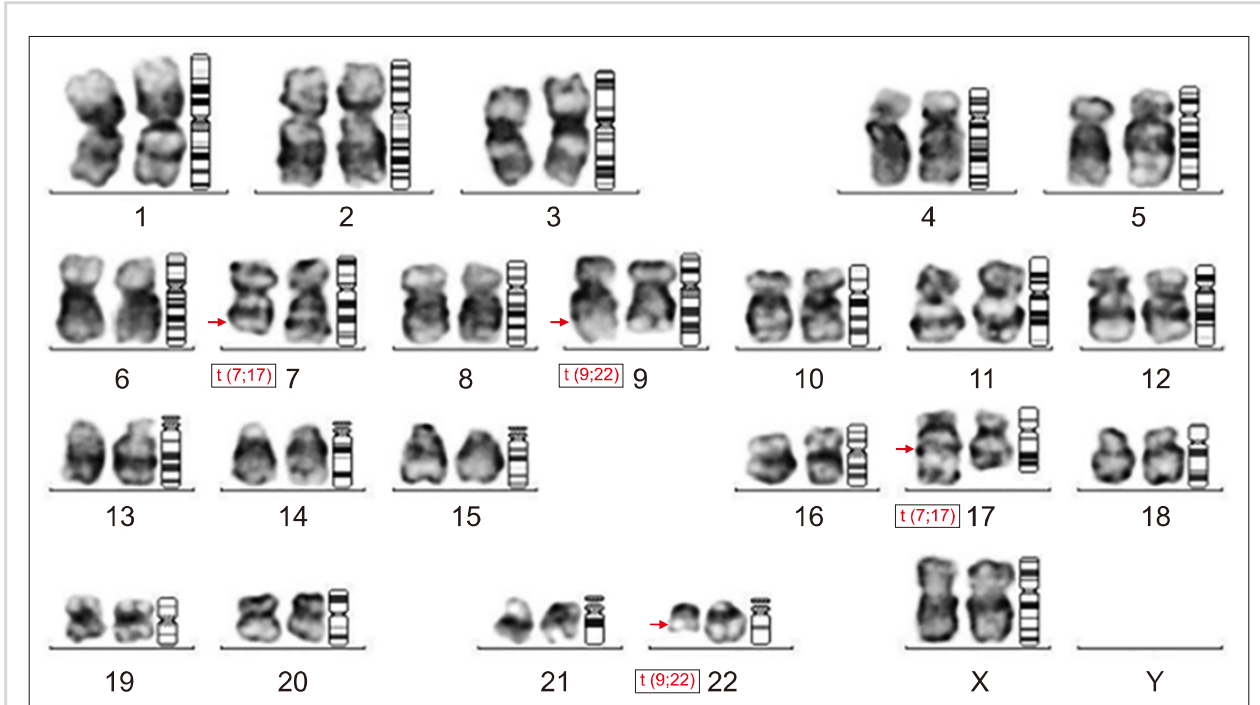

Fig. 2. Conventional cytogenetics, by $\mathrm{G}$ banding, of the bone marrow revealing Ph chromosome t $(9 ; 22)$ (q34:q11) and $t(7 ; 17)(q 22: q 21)$. 
at the time of clonal evolution in our case. Our case report described an entire course of disease in one patient, where $\mathrm{Ph}$-positive AML develops on the wheels of PNH in known cases of AA. BCR-ABL is considered a class I mutation in reference to $A M L$ and provides proliferation advantage to neoplastic clones [10]. Co-existing cytogenetic and molecular events may determine the preexisting illness in the form of AA, MDS, or de novo leukemia. In our case, the patient refused treatment or molecular workup that might have provided insight into the $\mathrm{BCR}-\mathrm{ABL}$ transcript type or other coexisting mutations.

The availability of SNP array karyotyping, exome sequencing, and next-generation sequencing in clinical labs has increased the momentum for the search for predictors of clonal evolution in AA. Cytogenetic abnormalities including -7/del 7q are associated with increased risk of progression to MDS/AML [12], while DNMT3A and ASXL1 mutations are good molecular predictors. These factors are also reported at variable frequencies in age-related clonal hematopoiesis. Hence, the application of this information in predicting prognosis and tailoring treatment is still not part of clinical practice. Our interesting case suggests that molecular and genetic events in AA, MDS, and AML are complex and overlapping.

Richa Juneja, Karthika Kundil Veetil, Gopila Gupta, Prasad Dange, Haraprasad Pati Department of Hematology, All India Institute of Medical Sciences, New Delhi, India

Correspondence to: Richa Juneja

Department of Hematology, All India Institute of Medical Sciences, Ansari Nagar East, New Delhi 110029, India E-mail: drrichajuneja@gmail.com

Received on Jan. 17, 2020; Revised on Jun. 4, 2020; Accepted on Jun. 11, 2020 https://doi.org/10.5045/br.2020.2020012

\section{Authors' Disclosures of Potential Conflicts of Interest}

No potential conflicts of interest relevant to this article were reported.

\section{REFERENCES}

1. Dameshek W. Riddle: what do aplastic anemia, paroxysmal nocturnal hemoglobinuria $(\mathrm{PNH})$ and "hypoplastic" leukemia have in common? Blood 1967;30:251-4.

2. Shimamura A. Aplastic anemia and clonal evolution: germ line and somatic genetics. Hematol Am Soc Hematol Educ Program 2016;2016:74-82.

3. Ogawa S. Clonal hematopoiesis in acquired aplastic anemia. Blood 2016;128:337-47.

4. Reboursiere E, Chantepie S, Gac AC, Reman O. Rare but authentic Philadelphia-positive acute myeloblastic leukemia: two case reports and a literature review of characteristics, treatment and outcome. Hematol Oncol Stem Cell Ther 2015; 8:28-33.
5. Swerdlow SH, Campo E, Harris NL, et al, eds. WHO classification of tumours of haematopoietic and lymphoid tissues. Revised 4th ed. Lyon, France: IARC Press, 2017.

6. Keung YK, Beaty M, Powell BL, Molnar I, Buss D, Pettenati M. Philadelphia chromosome positive myelodysplastic syndrome and acute myeloid leukemia-retrospective study and review of literature. Leuk Res 2004;28:579-86.

7. DeZern AE, Symons HJ, Resar LS, Borowitz MJ, Armanios MY, Brodsky RA. Detection of paroxysmal nocturnal hemoglobinuria clones to exclude inherited bone marrow failure syndromes. Eur J Haematol 2014;92:467-70.

8. Suzan F, Terré C, Garcia I, et al. Three cases of typical aplastic anaemia associated with a Philadelphia chromosome. $\mathrm{Br} \mathrm{J}$ Haematol 2001;112:385-7.

9. Breatnach F, Chessells JM, Greaves MF. The aplastic presentation of childhood leukaemia: a feature of common-ALL. Br J Haematol 1981;49:387-93

10. Neuendorff NR, Burmeister T, Dörken B, Westermann J. BCR-ABL-positive acute myeloid leukemia: a new entity? Analysis of clinical and molecular features. Ann Hematol 2016; 95:1211-21.

11. Fukunaga A, Sakoda H, Iwamoto Y, et al. Abrupt evolution of Philadelphia chromosome-positive acute myeloid leukemia in myelodysplastic syndrome. Eur J Haematol 2013;90:245-9.

12. Yoshizato T, Dumitriu B, Hosokawa K, et al. Somatic mutations and clonal hematopoiesis in aplastic anemia. N Engl J Med 2015;373:35-47.

\section{Response of multifocal acquired demyelinating sensorimotor neuropathy associated with atypical chronic lymphocytic leukemia to rituximab therapy}

TO THE EDITOR: Paraproteinemic peripheral neuropathy (PPN) refers to peripheral neuropathy (PN) associated with and considered to be mediated by a monoclonal protein in the serum+urine [1]. About $4 \%$ patients with PN have an associated monoclonal gammopathy (MG) [2]. An assemblage of MG $(<3 \mathrm{~g} / \mathrm{dL})$, small clonal population of bone marrow (BM) plasma cells (PCs) $(<10 \%)$, and absence of myeloma defining events defines monoclonal gammopathy of undetermined significance (MGUS). The prevalence of MGUS in general population is about $0.7 \%$, and it increases with age $(3.2 \%$ in $>50 \mathrm{yr}$ and $5.3 \%$ in $>70 \mathrm{yr}$ ) [3]. Therefore, both PN and MGUS may co-exist; ascribing pathogenicity of M-protein in such cases relies on demonstrating neurological improvement with treatment being targeted at the underlying clone secreting the implicated paraprotein. Recently, the term monoclonal gammopathy of clinical sig- 